\title{
ORDENAÇÕES PARCIAIS NOS CONJUNTOS DAS SOLUÇÕES DOS PROBLEMAS DE ALOCAÇÃO LINEAR E QUADRÁTICO
}

\author{
Maria Cristina Rangel * \\ Departamento de Informática - CT \\ Universidade Federal do Espírito Santo \\ Vitória - ES \\ crangel@,inf.ufes.br
}

\section{Nair Maria Maia de Abreu}

Programa de Engenharia de Produção - COPPE

Universidade Federal do Rio de Janeiro

Rio de Janeiro - RJ

$\underline{\text { nair@pep.ufrj.br }}$

* Corresponding author/autor para quem as correspondências devem ser encaminhadas

Recebido em 04/2002, aceito em 04/2003

\begin{abstract}
Resumo
O Problema Quadrático de Alocação, PQA, pode ser abordado através de uma relaxação na forma do Problema de Alocação Linear, PAL. Introduzimos um poset (conjunto parcialmente ordenado) no conjunto das soluções lineares que nos permite comparar também os custos das soluções do problema quadrático, sem o conhecimento prévio das matrizes que definem seus exemplares. Construímos um algoritmo polinomial capaz de determinar pares de permutações livremente comparáveis, conceito apresentado neste trabalho. Provamos um teorema que garante que os custos associados a tais permutações preservam a ordem dada pelo número de inversões das mesmas. Associando soluções do problema a permutações, testes empíricos são apresentados, visando a validação do número de inversões como um parâmetro de referência para a qualidade das soluções. Este trabalho é uma extensão do artigo [RA01] publicado nos anais do XXXIII SBPO, em CD-ROM, 1277-1287, Sobrapo, ILTC, 2001.
\end{abstract}

Palavras-chave: ordenação parcial; problema quadrático de alocação; problema de alocação linear.

\begin{abstract}
We present an approach to Quadratic Assignment Problem, QAP, via a linear relaxation, through the use of the Linear Assignment Problem, LAP. We define one ordering an LAP-solution-set that makes us compare QAP costs. From here, this poset, partial ordering set, considers coordinates of instances as free variables. We have managed to build a capable algorithm to determine freely comparable permutation pairs. A theorem shows that the order mentioned above is compatible to the one that involves inversion numbers of permutations. We can apply linear and quadratic solutions to permutations and empiric tests are presented to show that the inversion numbers can be used to measure the quality of QAP-solutions. This work is an extension of the article [RA01] published in electronic proceedings of SBPO XXXIII in CD-ROM, 1277-1287, Sobrapo, ILTC, 2001.
\end{abstract}

Keywords: partial ordination; quadratic assignment problem; linear assignment problem. 


\section{Introdução}

Em 1957, Koopmans e Beckmann, [KB57], idealizaram o Problema Quadrático de Alocação como um problema de layout, quando pretenderam instalar pares de $n$ fábricas a pares de $n$ locais, a custo mínimo, de modo que cada fábrica fosse atribuída a um único local e reciprocamente. Hoje, sabemos que o PQA é um dos mais difíceis problemas de Otimização Combinatória pertencente à classe NP-hard. Exemplares de ordem $n \geq 30$ desse problema já são considerados de grande porte e, portanto, intratáveis do ponto de vista computacional, se desejarmos resolvê-los otimamente. Em resposta a isto, considerável atenção vem sendo dispensada ao estudo do PQA por diversas abordagens, visando encontrar limites inferiores para auxiliar no desenvolvimento de algoritmos eficientes. Classificamos as diferentes abordagens em 3 grandes grupos:

(1) Formulações por Programação Inteira são dadas por programação binária, como a de [KB57], ou por Programação Mista, como as de [KB78], [FY83] e [PR96]. Nesta linha, diversos limites inferiores do tipo linear foram determinados, inclusive um dos mais antigos deles, conhecido por limite de Gilmore e Lawler e suas variações, [Gi62] e [La63];

(2) Formulações envolvendo Teoria das Matrizes, como é caso da formulação traço, devido a Edwards [Ed77] que resultou em limites espectrais, [Ed80]. Nesta categoria enquadramos também as mais recentes relaxações dadas por Programação Positiva Semidefinida, (SDP) que permitem cálculos de bons limites inferiores compatíveis com os espectrais, [Zh96] e [ZKRW98] e [An01]. Tais limites, apesar de melhores em qualidade que os lineares, demandam um tempo computacional considerável;

(3) Formulações por Matemática Discreta, de natureza puramente combinatória, como as devido à [Ab84], [Ra00] e [ABQG02]. Tais formulações definem o PQA associando permutações a soluções o que facilita caracterizar vizinhanças para busca local. Tais vizinhanças são muito utilizadas por meta-heurísticas. Nesta categoria destacam-se [LPR94], [Ta91], [Co90], [RAB00] e [GG02].

Além das referências mencionadas acima e daquelas que vamos citar no decorrer do trabalho, por vínculo direto ao estudo que desenvolvemos aqui, cabe recomendar o mais recente livro, dedicado exclusivamente ao Problema Quadrático de Alocação, devido a Eranda Çela, [Çe98], onde o leitor mais interessado poderá não só aprender mais sobre este problema, como também ter acesso a uma vasta bibliografia sobre ele. Na tentativa de completar a bibliografia até os dias de hoje, indicamos ainda alguns trabalhos mais recentes que, em geral, tratam de procedimentos e técnicas de resolução do PQA ou discutem as dificuldades por ele apresentadas, apontando a tendência mais atual das pesquisas, [GTD99], [AQB99], [RAB00], [AZ02], [ABLG02] e [Mi03].

Este trabalho aborda o Problema Quadrático de Alocação, PQA, por Matemática Discreta, apresentando o Problema de Alocação Linear, PAL, como uma relaxação do Problema Quadrático de Alocação. O conjunto de soluções do PAL, aqui representadas por permutações, contém todas as soluções do quadrático. Na segunda seção, introduzimos uma ordenação nos conjuntos das soluções do problema linear que é então induzida ao conjunto das soluções quadráticas, comparando seus respectivos custos, independentemente das matrizes que definem seus exemplares. Tal conjunto ordenado constitui o que chamamos de Poset da Ordenação Parcial Livre. Apresentamos, em seguida, um algoritmo polinomial para determinar pares de permutações livremente comparáveis, conceito introduzido 
em [Ab84]. Na Seção 4, apresentamos uma cadeia de grafos de comparabilidade para a cadeia dos posets dados na seção anterior. Propriedades para estes grafos são estabelecidas, visando um teorema que relaciona a ordenação parcial livre entre pares de permutações com o número de inversões das referidas permutações. Este teorema abre possibilidades para utilizarmos como parâmetro de avaliação da qualidade das soluções do PQA, o número de inversões das permutações a elas correspondentes. Finalizamos este trabalho utilizando alguns testes estatísticos na tentativa de investigar esta possibilidade.

\section{O Problema Quadrático de Alocação e sua Relaxação Linear}

Sejam duas matrizes $\mathrm{F}$ e $\mathrm{D}$, de dimensão $n \times n$, simétricas, com diagonal nula e cujas coordenadas reais são não-negativas. Resolver um exemplar do Problema Quadrático de Alocação, PQA(F,D), é achar o valor de

$$
Z\left(\varphi^{*}\right)=\min _{\varphi \in \Pi_{n}} \sum_{i<j} f_{i j} d_{\varphi(i) \varphi(j)}
$$

onde $\Pi_{n}$ é o conjunto de todas as permutações dos elementos de $\Omega^{\mathrm{n}}=\{1, \ldots n\}$. O valor resultante de (1) é o menor custo para uma atribuição em que pares de facilidades são alocadas a pares de localidades e reciprocamente. Trata-se de um problema de layout que pode ser representado por sobreposição de cliques de $n$ vértices, $\mathrm{K}_{\mathrm{F}}$ e $\mathrm{K}_{\mathrm{D}}$, onde a primeira possui suas arestas valoradas pelos fluxos das facilidades e a segunda, pelas distâncias das localidades. A sobreposição é definida por uma permutação dos vértices $\varphi \in \Pi_{n}$, cuja solução ótima é denotada por $\varphi^{*}$.

Considere o exemplar Nug5, devido a Nugent [NVR68], dada pelas matrizes simétricas:

$$
F=\left[\begin{array}{lllll}
0 & 5 & 2 & 4 & 1 \\
5 & 0 & 3 & 0 & 2 \\
2 & 3 & 0 & 0 & 0 \\
4 & 0 & 0 & 0 & 5 \\
1 & 2 & 0 & 5 & 0
\end{array}\right] \quad D=\left[\begin{array}{lllll}
0 & 1 & 1 & 2 & 3 \\
1 & 0 & 2 & 1 & 2 \\
1 & 2 & 0 & 1 & 2 \\
2 & 1 & 1 & 0 & 1 \\
3 & 2 & 2 & 1 & 0
\end{array}\right]
$$

Os elementos das matrizes $\mathrm{F}=\left[\mathrm{f}_{\mathrm{ij}}\right]$ e $\mathrm{D}=\left[\mathrm{d}_{\mathrm{k}}\right]$, que definem o $\mathrm{PQA}(\mathrm{F}, \mathrm{D})$, são armazenados em vetores-linhas (de mesmo nome) $\boldsymbol{F}=\left(f_{z}\right)$ e $\boldsymbol{D}=\left(d_{z}\right)$, ambos de dimensão $N=\mathrm{C}_{\mathrm{n}, 2}$, cujas coordenadas são dispostas segundo a bijeção

$$
\psi(i, j)=((i-1) n-i(i+1) / 2)+j,
$$

que associa o par $(i, j)$ a um número $z \in\{1, \ldots N\}$.

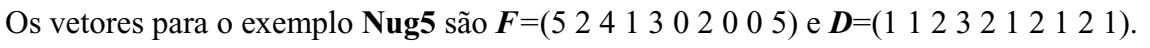

Considere a matriz $\mathrm{Q}=\boldsymbol{F}^{t} \boldsymbol{D}$, cujos coeficientes correspondem às parcelas da função objetivo (1), de modo que para cada $\varphi$, os coeficientes estejam na matriz Q. O Problema de Alocação Linear, PAL(Q), definido por

$$
Z\left(\xi^{*}\right)=\min _{\xi \in \Pi_{N}} \sum_{i=1}^{N} f_{i} d_{\xi(i)},
$$


onde $\Pi_{\mathrm{N}}$ é o conjunto de todas as permutações dos elementos de $\Omega^{\mathrm{N}}=\{1, \ldots N\}$, é uma relaxação linear do PQA(F,D), considerando-se que o conjunto das soluções lineares contém o das soluções quadráticas.

A partir de cada atribuição dos vértices de $\mathbf{K}_{\mathbf{F}}$ sobre $\mathbf{K}_{\mathbf{D}}$, representada por $\varphi$ em $\Pi_{n}$, determina-se, por intermédio de $\psi$ em (2), a correspondente sobreposição das arestas das referidas cliques, denotada por $\xi$. Em geral, dada uma solução $\xi$ do PAL(Q), nem sempre existe uma sobreposição de vértices das cliques compatível com a de arestas. Neste caso, diz-se que $\xi$ é uma solução não-viável para PQA(F,D). Se tal sobreposição $\varphi$ existir, deve obedecer a seguinte equação:

$$
f_{p} d_{\xi(p)}=f_{\psi^{-1}(p)} d_{\psi^{-1}(\xi(p))}=f_{i j} d_{k l},
$$

para $p=1, . . N ; \varphi(i)=k$ e $\varphi(j)=l$; onde $i, j, k, l=1, \ldots, n$. Assim, diz-se que $\xi$ é uma solução viável para PQA(F,D).

O custo da solução do PAL(Q) associado a $\xi \in \Pi_{\mathrm{N}}$ resulta do produto escalar,

$$
Z(\xi)=\langle F, \xi(D)\rangle,
$$

onde $\xi(D)=\left(d_{\xi(1)}, d_{\xi(2), \ldots .,} d_{\xi(N)}\right)$. No exemplo Nug5, seja uma solução de PAL(Q) dada por $\xi=\left(\begin{array}{llllllllll}1 & 2 & 3 & 4 & 5 & 6 & 7 & 8 & 9 & 10 \\ 2 & 1 & 4 & 6 & 7 & 9 & 3 & 5 & 8 & 10\end{array}\right)$. Podemos representar esta solução pela imagem de sua permutação correspondente, ou seja, $\xi=(214679358$ 10). Seu custo é então

$$
Z(\xi)=f_{1} d_{2}+f_{2} d_{1}+f_{3} d_{4}+f_{4} d_{6}+f_{5} d_{7}+f_{6} d_{9}+f_{7} d_{3}+f_{8} d_{5}+f_{9} d_{8}+f_{10} d_{10}=35 .
$$

Encontrar uma solução ótima para o PAL(Q) é muito fácil: basta associar a aresta de maior fluxo à de menor distância, sucessivamente, até que a aresta de menor fluxo seja associada à de maior distância. Desta forma, o custo mínimo é $Z^{*}=\left\langle F^{-}, D^{+}\right\rangle$, quando $\boldsymbol{F}^{-}$e $\boldsymbol{D}^{+}$são resultados da ordenação não-crescente e não-decrescente de $\boldsymbol{F}$ e $\boldsymbol{D}$, respectivamente, [HLP52]. O exemplar PAL $\left(\mathrm{Q}^{*}\right)$, onde $\mathrm{Q}^{*}=\left(\boldsymbol{F}^{-}\right)^{t} \boldsymbol{D}^{+}$possui o mesmo conjunto de soluções viáveis de $\mathrm{PAL}(\mathrm{Q})$ e, por conseguinte, a mesma solução ótima cujo custo é o traço de $\mathrm{Q}^{*}$, notado por $\operatorname{tr}\left(Q^{*}\right)$. Tal valor define um limite inferior para o $P Q A(F, D)$ e para o custo de qualquer outro exemplar obtido deste por troca de posições das coordenadas dos vetores de $\boldsymbol{F}$ e $\boldsymbol{D}$. Isto nos permite caracterizar uma classe de exemplares que chamamos de aparentados ao exemplar PQA $\left(F^{-}, D^{+}\right)$, por possuírem a mesma matriz $Q^{*}$. Formalmente, escrevemos:

$$
\operatorname{RelClass}\left(\mathbf{Q}^{*}\right)=\left\{\operatorname{PQA}(\mathrm{F}, \mathrm{D}) / \sigma(\boldsymbol{F})=\boldsymbol{F}^{-} \text {e } \tau(\boldsymbol{D})=\boldsymbol{D}^{+} \text {, para } \sigma \text { e } \tau \in \Pi_{\mathrm{N}}\right\} .
$$

Considere Nug5 proposto no exemplo anterior. Temos como vetores que definem o exemplar,

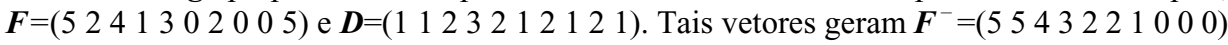

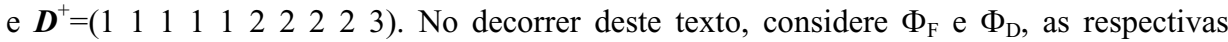
permutações auxiliares que conduzem $\boldsymbol{F}$ e $\boldsymbol{D}$ aos vetores ordenados $\boldsymbol{F}^{-}$e $\boldsymbol{D}^{+}$. Se $\rho \in \Pi_{\mathrm{N}}$ é uma solução do PAL(Q*), temos que a composta

$$
\xi=\Phi_{D}^{-1} \circ \rho \circ \Phi_{F}
$$

corresponde a uma solução do PAL(Q). Considerando-se os dados de Nug5, a permutação

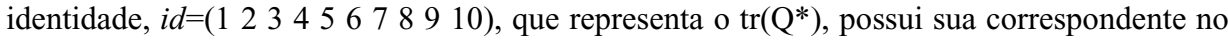




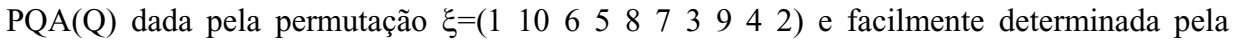

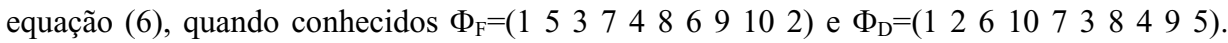
Veja a composição:

$\left(\begin{array}{cccccccccc}1 & 2 & 3 & 4 & 5 & 6 & 7 & 8 & 9 & 10 \\ 1 & 2 & 6 & 8 & 10 & 3 & 5 & 7 & 9 & 4\end{array}\right) \circ\left(\begin{array}{cccccccccc}1 & 2 & 3 & 4 & 5 & 6 & 7 & 8 & 9 & 10 \\ 1 & 2 & 3 & 4 & 5 & 6 & 7 & 8 & 9 & 10\end{array}\right) \circ\left(\begin{array}{llllllllll}1 & 2 & 3 & 4 & 5 & 6 & 7 & 8 & 9 & 10 \\ 1 & 5 & 3 & 7 & 4 & 8 & 6 & 9 & 10 & 2\end{array}\right)=\left(\begin{array}{cccccccccc}1 & 2 & 3 & 4 & 5 & 6 & 7 & 8 & 9 & 10 \\ 1 & 10 & 6 & 5 & 8 & 7 & 3 & 9 & 4 & 2\end{array}\right)$

Como saber se uma solução qualquer $\rho \in \Pi_{\mathrm{N}}$ do PAL(Q*) é viável para PQA(F,D)? Basta determinarmos $\varphi$ em $\Pi_{n}$, permutação dos vértices de uma clique sobre a outra, que seja compatível com a permutação das arestas $\rho$. De posse de $\Phi_{\mathrm{F}}$ e $\Phi_{\mathrm{D}}$ e da equação (6), se a série de igualdades a seguir for satisfeita,

$$
f_{r}^{-} d_{\rho(r)}^{+}=f_{\phi_{F}^{-1}(r)}^{-} d_{\phi_{D}^{-1}(\rho(r))}^{+}=f_{p} d_{\xi(p)}=f_{\psi^{-1}(p)} d_{\psi^{-1}(\xi(p))}=f_{i j} d_{k l},
$$

para $r=1, . . N ; \varphi(i)=k$ e $\varphi(j)=l$; onde $i, j, k, l=1, \ldots, n$, dizemos que $\rho \in \Pi_{\mathrm{N}}$ é solução viável para o PQA(F,D). Caso contrário esta solução é não viável. Pode-se observar que os dois primeiros termos da equação (7) são a aplicação das permutações $\Phi_{\mathrm{F}}$ e $\Phi_{\mathrm{D}}$ para encontrar os índices correspondentes às sobreposições das arestas antes das ordenações nas colunas de Q. Em seguida, as igualdades finais são completadas com a equação (4), que verifica a viabilidade de uma permutação $\xi \in \Pi_{\mathrm{N}}$, solução de $\operatorname{PAL}(\mathrm{Q})$, com relação ao problema quadrático.

\section{Uma Nova Ordenação Parcial no Conjunto dos Produtos Escalares}

Temos que o $\operatorname{tr}\left(\mathrm{Q}^{*}\right)$ associado a id em $\Pi_{\mathrm{N}}$ é o limite inferior para os custos de todos os exemplares do PQA(F,D) da família $\operatorname{RelClass}\left(\mathbf{Q}^{*}\right)$. Do mesmo modo, a soma dos elementos da diagonal secundária de $\mathrm{Q}^{*}$, que corresponde à permutação reversa, $r e v=(N, N-1, N-2, \ldots, 1)$ em $\Pi_{N}$, constitui um limite superior para este conjunto de custos. A comparação destes limites com qualquer outro custo do conjunto é independente das coordenadas das matrizes $\mathrm{F}$ e D que definem o PQA. Investigar a comparação dos custos de todos os pares possíveis de soluções dos exemplares em $\operatorname{RelClass}\left(\mathbf{Q}^{*}\right)$ resulta em determinar uma ordenação parcial, ou seja, um poset no conjunto dos custos desses problemas, cujo universo é constituído pelas soluções do PAL(Q). Este poset nos leva a um outro, no conjunto das permutações $\Pi_{N}$, dado que cada solução desses problemas pode ser associada a uma permutação. A construção dos referidos posets decorre do Teorema da Ordenação Parcial Livre, apresentado mais adiante e que notamos por TOPL.

Considere um par de permutações, $\rho_{1}$ e $\rho_{2}$ em $\Pi_{N}$, associadas às soluções do PAL( $\left.Q^{*}\right)$, cujos custos são dados pelos respectivos produtos escalares $Z\left(\rho_{l}\right)$ e $Z\left(\rho_{2}\right)$. Nosso objetivo é saber se $Z\left(\rho_{1}\right) \leq Z\left(\rho_{2}\right)$ ou $Z\left(\rho_{2}\right) \leq Z\left(\rho_{1}\right)$, sem o prévio conhecimento dos vetores envolvidos.

Da diferença dos produtos escalares temos

$$
Z\left(\rho_{1}\right)-Z\left(\rho_{2}\right)=\sum_{t=1}^{N} f_{t}^{-}\left(d_{\rho_{1}(t)}^{+}-d_{\rho_{2}(t)}^{+}\right)
$$

Para $t \in \Omega^{\mathrm{N}}=\{1, \ldots N\}$, seja fator-diferença o termo definido por $d_{\rho_{1}(t)}^{+}-d_{\rho_{2}(t)}^{+}$. O conjunto de índices do somatório em (8) é então dividido nos seguintes subconjuntos de $\Omega^{\mathrm{N}}=\mathrm{P}_{\mathrm{N}} \cup \mathrm{P}_{\mathrm{P}} \cup \mathrm{P}_{0}$, onde, 
- $\mathrm{P}_{\mathrm{N}}=\left\{t \in \Omega^{\mathrm{N}} / \rho_{1}(t)-\rho_{2}(t)<0\right\}$, índices dos fatores-diferença não-positivos de (8);

- $\mathrm{P}_{\mathrm{P}}=\left\{t^{\prime} \in \Omega^{\mathrm{N}} / \rho_{1}\left(t^{\prime}\right)-\rho_{2}\left(t^{\prime}\right)>0\right\}$, índices dos fatores-diferença não-negativos de (8) e;

- $\mathrm{P}_{0}=\left\{t^{\prime \prime} \in \Omega^{\mathrm{N}} / \rho_{1}\left(t^{\prime \prime}\right)-\rho_{2}\left(t^{\prime \prime}\right)=0\right\}$, índices dos fatores-diferença livremente nulos de (8).

Chamamos tal partição de partição ordenada dos índices em $\Omega^{\mathrm{N}}$.

Como conseqüência, o conjunto das parcelas do somatório na equação (8) é separado respectivamente, em parcelas não-positivas, não-negativas ou simplesmente nulas, que após a exclusão dessas últimas, chegamos a seguinte expressão para a diferença dos produtos escalares:

$$
Z\left(\rho_{1}\right)-Z\left(\rho_{2}\right)=\sum_{t \in P_{N}} f_{t}^{-}\left(d_{\rho_{1}(t)}^{+}-d_{\rho_{2}(t)}^{+}\right)+\sum_{t^{\prime} \in P_{P}} f_{t^{\prime}}^{-}\left(d_{\rho_{1}\left(t^{\prime}\right)}^{+}-d_{\rho_{2}\left(t^{\prime}\right)}^{+}\right) .
$$

A inserção de termos simétricos a cada fator-diferença nas parcelas da equação (9) nos leva a

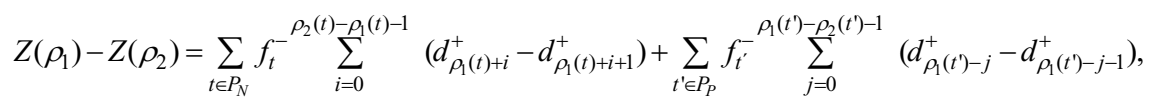

que chamamos de partição canônica da diferença dos produtos escalares $Z\left(\rho_{1}\right)$ e $Z\left(\rho_{2}\right)$. Analisando a equação (10), a seguinte proposição pode ser enunciada:

Proposição 1: Na partição canônica de $Z\left(\rho_{1}\right)-Z\left(\rho_{2}\right)$ temos que

$$
-\sum_{t \in P_{N}} \sum_{i=0}^{\rho_{2}(t)-\rho_{1}(t)-1}\left(d_{\rho_{1}(t)+i}^{+}-d_{\rho_{1}(t)+i+1}^{+}\right)=\sum_{t^{\prime} \in P_{P}} \sum_{j=0}^{\rho_{1}\left(t^{\prime}\right)-\rho_{2}\left(t^{\prime}\right)-1}\left(d_{\rho_{1}\left(t^{\prime}\right)-j}^{+}-d_{\rho_{1}\left(t^{\prime}\right)-j-1}^{+}\right) .
$$

Além disso, o valor absoluto de cada fator-diferença do primeiro membro corresponde a um fator-diferença igual no segundo membro e reciprocamente.

Prova: Considere $\rho_{1}$ e $\rho_{2}$ em $\Pi_{\mathrm{N}}$. Temos que

$$
\sum_{t \in \Omega_{N}}\left(d_{\rho_{1}(t)}^{+}-d_{\rho_{2}(t)}^{+}\right)=0 .
$$

Utilizando os subconjuntos de índices da união ordenada, $\mathrm{P}_{\mathrm{N}} \cup \mathrm{P}_{\mathrm{P}} \cup \mathrm{P}_{0}$, chegamos a

$$
\sum_{t \in P_{N}}\left(d_{\rho_{1}(t)}^{+}-d_{\rho_{2}(t)}^{+}\right)+\sum_{t^{\prime} \in P_{P}}\left(d_{\rho_{1}\left(t^{\prime}\right)}^{+}-d_{\rho_{2}\left(t^{\prime}\right)}^{+}\right)=0 .
$$

Introduzindo-se os termos simétricos, o valor da expressão acima não se altera e chegamos a equação da Proposição 1.

Para demonstração da segunda parte desta proposição, é fácil ver que $\forall t \in \mathrm{P}_{\mathrm{N}}$ e $i \in\left\{0, \ldots, \rho_{2}(t)-\rho_{1}(t)-1\right\} \quad$ existe um $t^{\prime} \in \mathrm{P}_{\mathrm{P}} \quad \mathrm{e} \quad j \in\left\{0, \ldots, \rho_{1}\left(t^{\prime}\right)-\rho_{2}\left(t^{\prime}\right)-1\right\} \quad$ tal que $\rho_{1}(t)+1=\rho_{1}\left(t^{\prime}\right)-j-1$ e $\rho_{1}(t)+i+1=\rho_{1}\left(t^{\prime}\right)-j$, resultando em

$$
-\left(d_{\rho_{1}(t)+i}^{+}-d_{\rho_{1}(t)+i+1}^{+}\right)=\left(d_{\rho_{1}\left(t^{\prime}\right)-j}^{+}-d_{\rho_{1}\left(t^{\prime}\right)-j-1}^{+}\right) .
$$

Definimos $N_{t}^{i}=f_{t}\left(d_{\rho_{1}(t)+i}^{+}-d_{\rho_{1}(t)+i+1}^{+}\right)$, com $t \in \mathrm{P}_{\mathrm{N}}$, para as parcelas da partição canônica cujos valores são não-positivos; $P_{t^{\prime}}^{j}=f_{t^{\prime}}\left(d_{\rho_{1}\left(t^{\prime}\right)-j}^{+}-d_{\rho_{1}\left(t^{\prime}\right)-j-1}^{+}\right)$, com $t^{\prime} \in \mathrm{P}_{\mathrm{P}}$, para as parcelas cujos valores são não-negativos, onde $i=0, \ldots, \rho_{2}(t)-\rho_{1}(t)-1$ e $j=0, \ldots, \rho_{1}\left(t^{\prime}\right)-\rho_{2}\left(t^{\prime}\right)-1$. 
De acordo com a proposição anterior, chamamos de fatores-diferença simétricos aqueles que são iguais em valor absoluto e cujos índices obedecem à seguinte relação:

$$
\rho_{1}(t)+1=\rho_{1}\left(t^{\prime}\right)-j-1 \text { e } \rho_{1}(t)+i+1=\rho_{1}\left(t^{\prime}\right)-j .
$$

Proposição 2: Existe, pelo menos, uma bijeção $\beta$ que associa cada parcela $N_{t}^{i}$, onde $i=0, \ldots, \rho_{2}(t)-\rho_{1}(t)-1$, a uma parcela $P_{t^{\prime}}^{j}$, onde $j=0, \ldots, \rho_{1}\left(t^{\prime}\right)-\rho_{2}\left(t^{\prime}\right)-1$, tal que seus respectivos fatores-diferença sejam simétricos. Para cada $t \in \mathrm{P}_{\mathrm{N}}$ e $t^{\prime} \in \mathrm{P}_{\mathrm{P}}$ e para cada uma das bijeções que satisfaça $\beta\left(N_{t}^{i}\right)=P_{t^{\prime}}^{j}$, tem-se que $\beta$ induz os pares ordenados $\left(t, t^{\prime}\right)$. Além disso, para $f_{t}^{-}$e $f^{-}{ }^{\prime}$, sendo, respectivamente, as $t$-ésima e $t^{\prime}$-ésima coordenadas do vetor $\boldsymbol{F}^{-}$ referentes ao par $\left(t, t^{\prime}\right)$, a partição canônica de $\boldsymbol{Z}\left(\rho_{1}\right)-\boldsymbol{Z}\left(\rho_{2}\right)$ resulta em

$$
Z\left(\rho_{1}\right)-Z\left(\rho_{2}\right)=\sum_{t \in P_{N}} \sum_{i=0}^{\rho_{2}(t)-\rho_{1}(t)-1}\left(f_{t}^{-}-f_{t^{-}}^{-}\right)\left(d_{\rho_{1}(t)+i}^{+}-d_{\rho_{1}(t)+i+1}^{+}\right) .
$$

Prova: Basta construir uma bijeção $\beta$ tal que $\beta\left(N_{t}^{i}\right)=P_{t^{\prime}}^{j}, \forall t \in \mathrm{P}_{\mathrm{N}}, i=0, \ldots, \rho_{2}(t)-\rho_{1}(t)-1$, $\forall t^{\prime} \in \mathrm{P}_{\mathrm{P}}$ e $j=0, \ldots, \rho_{1}\left(t^{\prime}\right)-\rho_{2}\left(t^{\prime}\right)-1$. A existência da parcela $P_{t^{\prime}}^{j}$ é garantida pela Proposição 1 . Dado que os fatores-diferença são simétricos, colocando-os em evidência, obtemos facilmente a expressão da Proposição 2. No momento da construção de $\beta$ os pares ordenados $\left(t, t^{\prime}\right), t \in \mathrm{P}_{\mathrm{N}}$ e $t^{\prime} \in \mathrm{P}_{\mathrm{P}}$, são identificados.

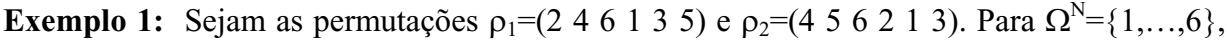
temos $\mathrm{P}_{\mathrm{N}}=\{1,2,4\}, \mathrm{P}_{\mathrm{P}}=\{5,6\}$ e $\mathrm{P}_{0}=\{3\}$. Calculando-se a diferença $Z\left(\rho_{1}\right)-Z\left(\rho_{2}\right)$ dos produtos escalares, após descartarmos o termo livremente nulo, relativo a $P_{0}$, sendo que $\rho_{1}(3)=\rho_{2}(3)=6$ e $d_{\rho_{1}(3)}^{+}-d_{\rho_{2}(3)}^{+}=d_{6}^{+}-d_{6}^{+}=0$, chegamos a

$$
Z\left(\rho_{1}\right)-Z\left(\rho_{2}\right)=f_{1}^{-}\left(d_{2}^{+}-d_{4}^{+}\right)+f_{2}^{-}\left(d_{4}^{+}-d_{5}^{+}\right)+f_{4}^{-}\left(d_{1}^{+}-d_{2}^{+}\right)+f_{5}^{-}\left(d_{3}^{+}-d_{1}^{+}\right)+f_{6}^{-}\left(d_{5}^{+}-d_{3}^{+}\right) .
$$

Inserindo os termos simétricos em cada fator-diferença e reorganizando-os de modo que fatores-diferença simétricos sejam identificados tem-se:

$$
\begin{aligned}
Z\left(\rho_{1}\right)-Z\left(\rho_{2}\right)= & f_{1}^{-}\left(d_{2}^{+}-d_{3}^{+}\right)+f_{1}^{-}\left(d_{3}^{+}-d_{4}^{+}\right)+f_{2}^{-}\left(d_{4}^{+}-d_{5}^{+}\right)+f_{4}^{-}\left(d_{1}^{+}-d_{2}^{+}\right)+ \\
& f_{5}^{-}\left(d_{3}^{+}-d_{2}^{+}\right)+f_{5}^{-}\left(d_{2}^{+}-d_{1}^{+}\right)+f_{6}^{-}\left(d_{5}^{+}-d_{4}^{+}\right)+f_{6}^{-}\left(d_{4}^{+}-d_{3}^{+}\right) .
\end{aligned}
$$

Neste momento, já podemos escrever a partição canônica de $Z\left(\rho_{l}\right)-Z\left(\rho_{2}\right)$ de acordo com a expressão da Proposição 2.

$$
\begin{aligned}
Z\left(\rho_{1}\right)-Z\left(\rho_{2}\right)= & \left(f_{1}^{-}-f_{5}^{-}\right)\left(d_{2}^{+}-d_{3}^{+}\right)+\left(f_{1}^{-}-f_{6}^{-}\right)\left(d_{3}^{+}-d_{4}^{+}\right)+\left(f_{2}^{-}-f_{6}^{-}\right)\left(d_{4}^{+}-d_{5}^{+}\right)+ \\
& \left(f_{4}^{-}-f_{5}^{-}\right)\left(d_{1}^{+}-d_{2}^{+}\right) .
\end{aligned}
$$

Então, o conjunto dos pares ordenados $\left(t, t^{\prime}\right)$, com $t \in \mathrm{P}_{\mathrm{N}}$ e $t^{\prime} \in \mathrm{P}_{\mathrm{P}}$, induzidos pela bijeção $\beta$ é $\{(1,5),(1,6),(2,6),(4,5)\}$.

Considerando-se vetores $\boldsymbol{F}^{-}$e $\boldsymbol{D}^{+}$de coordenadas reais e não-negativas, seja o seguinte conjunto de produtos escalares:

$$
C(Z(\rho))=\left\{Z(\rho)=\left\langle F^{-}, \rho\left(D^{+}\right)\right\rangle / \rho \in \Pi_{N}\right\} .
$$


Dizemos que $Z\left(\rho_{l}\right)$ e $Z\left(\rho_{2}\right)$ são livremente comparáveis, se e somente se, uma das duas condições for satisfeita: $Z\left(\rho_{1}\right) \leq Z\left(\rho_{2}\right)$ ou $Z\left(\rho_{2}\right) \leq Z\left(\rho_{1}\right)$ independentemente das coordenadas dos vetores $\boldsymbol{F}^{-}$e $\boldsymbol{D}^{+}$. Tais relações são denotadas por $Z\left(\rho_{1}\right) \leq_{l} Z\left(\rho_{2}\right)$ ou $Z\left(\rho_{2}\right) \leq_{l} Z\left(\rho_{1}\right)$.

Teorema da Ordenação Parcial Livre (TOPL): Temos $Z\left(\rho_{1}\right) \leq_{l} Z\left(\rho_{2}\right)$ (ou $\left.Z\left(\rho_{2}\right) \leq_{l} Z\left(\rho_{1}\right)\right)$ se e somente se, existir uma bijeção $\beta$, tal que $\beta\left(N_{t}^{i}\right)=P_{t^{\prime}}^{j}$ e ainda se $\beta$ induzir os pares ordenados $\left(t, t^{\prime}\right), t \in \mathrm{P}_{\mathrm{N}}$ e $t^{\prime} \in \mathrm{P}_{\mathrm{P}}$, de modo que se tenha $t<t^{\prime}$ (ou $\left.t^{\prime}<t\right), \forall t \in \mathrm{P}_{\mathrm{N}}$ e $\forall t^{\prime} \in \mathrm{P}_{\mathrm{P}}$.

Prova: $\left(\Rightarrow\right.$ ) Segue diretamente dos vetores $\boldsymbol{F}^{-}$e $\boldsymbol{D}^{+}$serem ordenados em ordem nãocrescente e não-decrescente, respectivamente, e após aplicação da Proposição 2.

$(\Leftarrow)$ Considere $Z\left(\rho_{1}\right) \leq_{l} Z\left(\rho_{2}\right)$. Da Proposição 1 , temos a existência de uma bijeção $\beta$ que induz os pares ordenados $\left(t, t^{\prime}\right)$, tais que $t<t^{\prime}, \forall t \in \mathrm{P}_{\mathrm{N}}$ e $\forall t^{\prime} \in \mathrm{P}_{\mathrm{P}}$. Suponhamos a possibilidade de termos, ao mesmo tempo, $Z\left(\rho_{1}\right) \leq_{l} Z\left(\rho_{2}\right)$ e um par ordenado $\left(t^{*}, t^{*}\right)$ induzido por alguma $\beta$, com $t^{*}<t^{*}$ para $t^{*} \in \mathrm{P}_{\mathrm{N}}$ e $t^{*} \in \mathrm{P}_{\mathrm{P}}$. Da Proposição 2 , temos:

$$
Z\left(\rho_{1}\right)-Z\left(\rho_{2}\right)=\sum_{t \in P_{N}} \sum_{i=0}^{\rho_{2}(t)-\rho_{1}(t)-1}\left(f_{t}^{-}-f_{t^{\prime}}^{-}\right)\left(d_{\rho_{1}(t)+i}^{+}-d_{\rho_{1}(t)+i+1}^{+}\right) \leq 0 .
$$

Considerando-se a ordenação de $\boldsymbol{F}^{-}$temos $\left(f_{t^{*}}^{-}-f_{t^{-} *}^{-}\right) \leq 0$ e da ordenação de $\boldsymbol{D}^{+}$, $\left(d_{\rho_{1}(t)+i}^{+}-d_{\rho_{1}(t)+i+1}^{+}\right) \leq 0$. Agora, considere um valor para L dado pela expressão

$$
L=\sum_{i=0}^{\rho_{2}\left(t^{*}\right)-\rho_{1}\left(t^{*}\right)-1}\left(f_{t^{*}}^{-}-f_{t^{\prime} *}^{-}\right)\left(d_{\rho_{1}(t *)+i}^{+}-d_{\rho_{1}(t *)+i+1}^{+}\right) \geq 0 .
$$

Tomemos um caso do PQA tal que L seja suficientemente grande para termos

$$
L \geq \sum_{t \in P_{N}-\left\{t^{*}\right\}} \sum_{i=0}^{\rho_{2}(t)-\rho_{1} 0^{-1}}\left(f_{t}^{-}-f_{t^{-}}^{-}\right)\left(d_{\rho_{1}(t)+i}^{+}-d_{\rho_{1}(t)+i+1}^{+}\right),
$$

para todo par $\left(t, t^{\prime}\right)$, induzido da bijeção $\beta$. Para esse caso, ocorre a contradição, dado que $Z(\rho)-Z\left(\rho_{2}\right) \leq 0$.

A ordem parcial definida pelo TOPL introduz o poset $\left(C(Z(\rho)), \leq_{l}\right)$ no conjunto dos custos do Problema de Alocação Linear cujos elementos, por ela relacionados, chamamos pares livremente comparáveis. A partir da relação

$$
\rho_{i} \leq_{l} \rho_{j} \text {, se e somente se, } Z\left(\rho_{i}\right) \leq_{l} Z\left(\rho_{j}\right), i, j=1, . . N \text { ! }
$$

um novo poset é induzido no conjunto das permutações de $\Pi_{\mathrm{N}}$, denotado por $\left(\Pi_{N}, \leq_{l}\right)$. Dizemos então que permutações livremente comparáveis são aquelas que satisfazem a relação (15). O grafo de comparabilidade do poset $\left(\Pi_{N}, \leq_{l}\right)$ é notado por $G_{L i v}=\left(\Pi_{\mathrm{N}}, \mathrm{M}\right)$, onde $\mathbf{M}=\left\{\left(\rho_{i}, \rho_{j}\right) / \rho_{i} \leq_{l} \rho_{j}, i, j=\{1, \ldots, N !\}\right\}$. Dizemos que a ordenação é livre pelo fato de envolver permutações cujos custos são comparáveis considerando-se as coordenadas dos vetores $\boldsymbol{F}^{-}$e $\boldsymbol{D}^{+}$como variáveis livres. 
Para verificar se pares de permutações são livremente comparáveis o algoritmo AlgBijeção, constrói uma bijeção canônica, a partir da Proposição 2, que denotamos de $\beta_{\mathrm{c}}$. Se esta bijeção determinar que as permutações são ou não livremente comparáveis, não há necessidade de se buscar outra. Este algoritmo é importante, pois evita a enumeração das possíveis bijeções, visto que os fatores-diferença podem se repetir. As listas ListaN e ListaP armazenam os índices das parcelas $N_{t}^{i}$ e $P_{t}^{j}$, respectivamente, utilizando os conjuntos $\mathrm{P}_{\mathrm{N}}$ e $\mathrm{P}_{\mathrm{P}}$. Define-se como par de elementos simétricos nas listas ListaN e ListaP aqueles que obedece às relações $\rho_{1}(t)+i=\rho_{1}\left(t^{\prime}\right)-j-1$ e $\rho_{1}(t)+i+1=\rho_{1}\left(t^{\prime}\right)-j$, para $t \in \mathrm{P}_{\mathrm{N}}$ e $t^{\prime} \in \mathrm{P}_{\mathrm{p}}$, onde $i=0, \ldots, \rho_{2}(t)-\rho_{1}(t)-1$ e $j=0, \ldots, \rho_{1}\left(t^{\prime}\right)-\rho_{2}\left(t^{\prime}\right)-1$. A lista chamada ListaPares guarda os pares ordenados $\left(t, t^{\prime}\right)$ de tais elementos simétricos.

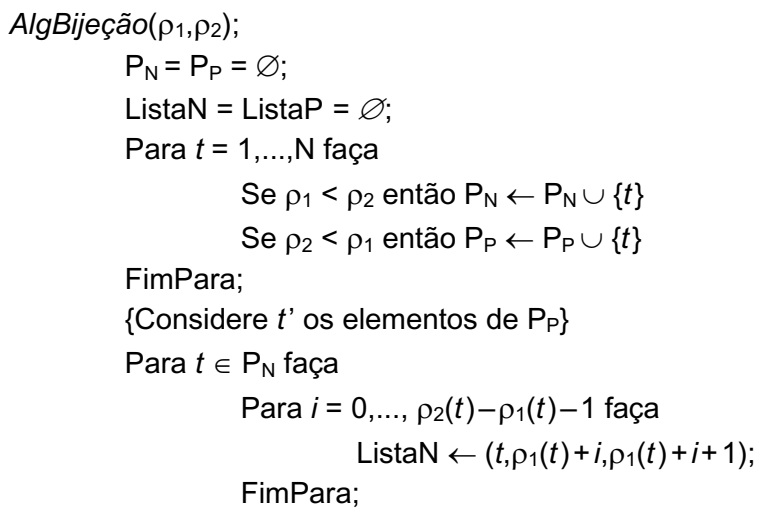

FimPara;

Para $t^{\prime} \in \mathrm{P}_{\mathrm{p}}$ faça

$$
\begin{aligned}
\text { Para } j= & 0, \ldots, \rho_{1}\left(t^{\prime}\right)-\rho_{2}\left(t^{\prime}\right)-1 \text { faça } \\
& \text { ListaP } \leftarrow\left(t^{\prime}, \rho_{1}\left(t^{\prime}\right)-j, \rho_{1}\left(t^{\prime}\right)-j-1\right) ;
\end{aligned}
$$

FimPara;

FimPara;

Para $k=1$ to $\mid$ ListaN| faça

Buscar em ListaP dentre os elementos não marcados o primeiro simétrico de ListaN[k];

Marcar elemento encontrado;

Formar o par $\left(t, t^{\prime}\right)$;

ListaPares $\leftarrow\left(t, t^{\prime}\right)$;

FimPara;

Se os pares ordenados $\left(t, t^{\prime}\right) \in \mathrm{ListaP}$, tenha $t<t^{\prime}$ (ou $t^{\prime}<t$ ), $\forall t \in \mathrm{P}_{\mathrm{N}}$ e $\forall t^{\prime} \in \mathrm{P}_{\mathrm{P}}$ então as permutações são livremente comparáveis.

Fim AlgBijeção;

Teorema 3.1: A complexidade do algoritmo AlgBijeção é de ordem $O\left(N^{4}\right)$.

Prova: O pior caso acontece quando o procedimento percorre a ListaP em busca do simétrico, para cada elemento da ListaN. Ambas possuem, no máximo, $N^{2} / 4$ elementos, quando $N$ é par, e $\left(N^{2}-1\right) / 4$ elementos quando, $N$ é ímpar. 


\section{Grafos de Comparabilidade e o Teorema das Inversões}

Seja a relação < definida em $\Pi_{\mathrm{N}}$ da seguinte maneira: " $\rho_{s}<\rho_{r}$ se existe $i \in \Omega^{\mathrm{N}}$, tal que $\rho_{s}(i)<\rho_{r}(i) ; \rho_{s}(i)<\rho_{r}(i+1) ; \rho_{s}(i+1)<\rho_{r}(i)$ e $\forall k$ em $\Omega^{N}, k=\{1, \ldots i-1, i+2, \ldots N\}, \rho_{s}(k)=\rho_{r}(k)$ ”. A relação transitiva de $<$ determina o poset $\left(\Pi_{N},<\right)$ que junto com as operações de join e meet caracterizam o conhecido Reticulado das Permutações, cujo Diagrama de Hasse é dado por um grafo chamado Permutaedro [VRA95]. Uma inversão em $\rho$, dada pelo seu par de inversões $(\rho(\mathbf{i}), \rho(\mathbf{j}))$ é tal que se $i<j$ então $\rho(j)<\rho(i)$, para todo $i, j \in \Omega^{\mathrm{N}}$. A cardinalidade do conjunto $\mathfrak{I}(\rho)$, formado por tais pares é o número de inversões de $\rho$, denotado por $\mathbf{I}(\mathcal{I}(\rho) \mathbf{I}$. Seja $W=\left\{\left(\rho_{\mathrm{r}}, \rho_{\mathrm{s}}\right) \in \Pi_{\mathrm{N}} \mathrm{x} \Pi_{\mathrm{N}} / \rho_{\mathrm{s}}\right.$ é obtida de $\rho_{\mathrm{r}}$ por troca de elementos adjacentes com $\left.\left|\mathfrak{I}\left(\rho_{\mathrm{s}}\right)\right|-\left|\mathfrak{I}\left(\rho_{\mathrm{r}}\right)\right|=1\right\}$. O grafo $G_{I n v}=\left(\Pi_{\mathrm{N}}, \mathrm{W}\right)$ é o grafo de comparabilidade de $\left(\Pi_{N},<\right)$, também chamado de grafo das inversões. Desta forma, o Permutaedro realiza graficamente $G_{I n v}$ e seus vértices são dispostos em níveis pelo número de inversões de suas permutações. A construção desse grafo se inicia no nível 0 , pela $i d=(1,2, \ldots, N)$. Efetuando-se as trocas dos elementos adjacentes, nível a nível, chega-se à permutação $r e v=(N, N-1, \ldots, 1)$, última permutação gerada no nível $N(N-1) / 2+1$.

Lema 4.1: Sejam $\rho_{\mathrm{i}}$ e $\rho_{\mathrm{j}}$ em $\Pi_{\mathrm{N}}$. Se $\left(\rho_{\mathrm{i}}, \rho_{\mathrm{j}}\right) \in \mathrm{W}$ então $Z\left(\rho_{i}\right) \leq_{l} Z\left(\rho_{j}\right)$.

Prova: A prova deste lema segue diretamente das definições dos conjuntos $\mathrm{P}_{\mathrm{N}}$ e $\mathrm{P}_{\mathrm{P}}$ e da aplicação da Proposição 2.

O grafo $G_{I n v}$ é um grafo parcial de $G_{L i v}$, grafo de comparabilidade do poset $\left(\Pi_{N}, \leq_{l}\right)$, que representa as permutações livremente comparáveis. No grafo fecho transitivo de $G_{I n v}, \overline{G_{I n v}}$, a relação de ordem de $\leq_{l}$ é preservada em seus vértices que são ligados por um caminho em W. O lema a seguir, relaciona a variação dos custos com o número das inversões das permutações a eles correspondentes.

Lema 4.2: Sejam $\rho_{\mathrm{i}}$ e $\rho_{\mathrm{j}}$ vértices do grafo $G_{I n v}$ ligados por um caminho de arcos em W. Tem-se $\left|\mathfrak{I}\left(\rho_{i}\right)\right|<\left|\mathfrak{I}\left(\rho_{j}\right)\right|$, se e somente se, $Z\left(\rho_{i}\right) \leq{ }_{l} Z\left(\rho_{j}\right)$.

Prova: Uma indução sobre o número de arcos do caminho entre os vértices é considerada. Vamos supor, inicialmente, que o caminho entre $\rho_{\mathrm{i}}$ e $\rho_{\mathrm{j}}$ tenha comprimento 1 , isto é, $\mid \mathfrak{T}\left(\rho_{j}\right) /-/ \mathfrak{T}\left(\rho_{i}\right) /=1$. Logo, do Lema 4.1, como $\left(\rho_{\mathrm{i}}, \rho_{\mathrm{j}}\right) \in \mathrm{W}$ então $Z\left(\rho_{i}\right) \leq_{l} Z\left(\rho_{j}\right)$. Suponhamos que o resultado seja válido para todos os caminhos de comprimento $(m-1)$ de $\rho_{\mathrm{i}}$ e $\rho_{\mathrm{j}}$. Para facilitar a notação, considere $\rho_{\mathrm{i}}=\rho_{1}$ e $\rho_{\mathrm{j}}=\rho_{\mathrm{m}}$. Pela hipótese de indução segue-se que $Z\left(\rho_{1}\right) \leq_{l} Z\left(\rho_{m}\right)$. Tomemos o $m$-ésimo arco do caminho entre $\rho_{\mathrm{m}}$ e $\rho_{\mathrm{m}+1}$ tal que $\left(\rho_{\mathrm{m}}, \rho_{\mathrm{m}+1}\right) \in \mathrm{W}$. Analogamente, tem-se que a relação $Z\left(\rho_{m}\right) \leq_{l} Z\left(\rho_{m+1}\right)$. Por transitividade chegamos a $Z\left(\rho_{1}\right) \leq{ }_{l} Z\left(\rho_{m+1}\right)$. Retomando a notação original segue-se que $\left|\mathfrak{T}\left(\rho_{i}\right)\right|<\left|\mathfrak{T}\left(\rho_{j}\right)\right|$, se e somente se, $Z\left(\rho_{i}\right) \leq_{l} Z\left(\rho_{j}\right)$.

O nosso objetivo é estender este resultado para o grafo $G_{L i v}$, atingindo assim todas as permutações livremente comparáveis. Para isso, definamos o conjunto de $\operatorname{arcos} \mathrm{W}^{\prime}=\left\{\left(\rho_{\mathrm{i}}, \rho_{\mathrm{j}}\right) /\right.$ $Z\left(\rho_{i}\right) \leq_{l} Z\left(\rho_{j}\right)$ e $\left.\left|\mathfrak{I}\left(\rho_{\mathrm{i}}\right)\right|-\left|\mathfrak{J}\left(\rho_{\mathrm{j}}\right)\right|=1\right\}$, que contém $\mathrm{W}$, para constituir $G^{\prime}{ }_{\text {Inv }}=\left(\Pi_{\mathrm{N}}, \mathrm{W}^{\prime}\right)$ que é um grafo parcial de $G_{L i v}$. Portanto, temos a cadeia de grafos $G_{I n v} \subseteq G_{I n v} \subseteq G_{L i v}$. 
Lema 4.3: Sejam $\rho_{\mathrm{i}}$ e $\rho_{\mathrm{j}}$ em $\Pi_{\mathrm{N}}$. Se $\left(\rho_{\mathrm{i}}, \rho_{\mathrm{j}}\right) \in \mathrm{W}$ ' então $Z\left(\rho_{i}\right) \leq_{l} Z\left(\rho_{j}\right)$.

Prova: Os $\operatorname{arcos}\left(\rho_{\mathrm{i}}, \rho_{\mathrm{j}}\right) \in \mathrm{W}$ ' são compostos de permutações que diferem entre si por dois elementos, assegurando que são livremente comparáveis [Ran00]. Isto inclui os arcos pertencentes a W, resultantes de trocas de elementos adjacentes da permutação.

Desejamos provar que a ordem livremente comparável entre os produtos escalares $Z\left(\rho_{i}\right)$ e $Z\left(\rho_{j}\right)$ preserva a ordem natural dada pelo número de inversões de $\rho_{\mathrm{i}}$ e $\rho_{\mathrm{j}}$. Para tanto, precisamos ainda do próximo lema que constrói o fecho transitivo do grafo $G_{I n v}$, denotado por $\overline{G_{I n v}^{\prime}}$ e que funciona como suporte para o Teorema das Inversões.

Lema 4.4: Sejam $\rho_{\mathrm{i}}$ e $\rho_{\mathrm{j}}$ vértices do grafo $G_{\text {Inv }}^{\prime}$, ligados por um caminho de arcos em $\mathrm{W}$ '. Tem-se $\left|\mathfrak{T}\left(\rho_{i}\right)\right|<\left|\mathfrak{T}\left(\rho_{j}\right)\right|$ se e somente se $Z\left(\rho_{i}\right) \leq_{l} Z\left(\rho_{j}\right)$.

A demonstração deste lema é análoga ao do Lema 4.2, considerando, neste caso, os arcos pertencentes a $\mathrm{W}^{\prime}$.

A Figura 4.1 ilustra o $G^{\prime}{ }_{I n v}$ de ordem $N=4$. Em destaque estão os arcos de W' - W. Este grafo não representa o conjunto das soluções de nenhum exemplar do PQA, pois não existe um natural $n$ tal que $\mathrm{C}_{\mathrm{n}, 2}=4$. A menor ordem significativa para algum caso desse problema tem dimensão $n=4$, o que apesar de muito pequeno, já nos impossibilita uma representação gráfica completa, dado que $N=6$ e o grafo $G_{\text {Inv }}{ }^{\prime}$ teria 720 nós.

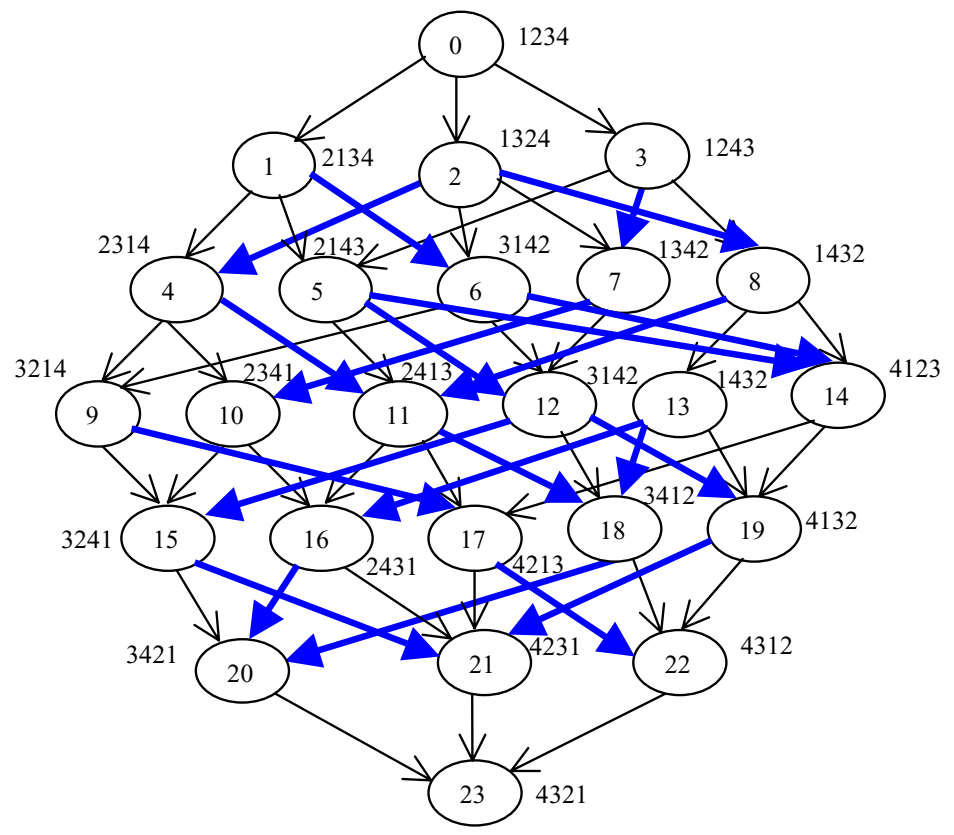

Figura 4.1 - Grafo $G_{\text {Inv }}^{\prime}$ para $N=4$ e destaque para os $\operatorname{arcos}\left(\mathrm{W}^{\prime}-\mathrm{W}\right)$. 
O Teorema das Inversões, a seguir apresentado, mostra que todo arco de $G_{L i v}$ está em $\overline{G_{I n v}^{\prime}}$. Desta forma $G_{L i v}$ é também o grafo de comparabilidade do poset de permutações livremente comparáveis.

Teorema das Inversões: Sejam $\overline{G_{I n v}^{\prime}}$ o fecho transitivo de $G_{I n v}^{\prime}=\left(\Pi_{\mathrm{N}}, \mathrm{W}^{\prime}\right)$ e $G_{L i v}=\left(\Pi_{\mathrm{N}}, \mathrm{M}\right)$ o grafo de comparabilidade do poset $\left(\Pi_{N}, \leq_{l}\right)$. Tem-se que $\overline{G_{I n v}^{\prime}}=G_{L i v}$.

Prova: $(\Rightarrow) \overline{G_{I n v}^{\prime}}$ é um subgrafo de $G_{L i v}$ pois possui o mesmo conjunto de nós e pelo Lema 4.4 , tem-se que $\mathrm{W}^{\prime} \subseteq \mathrm{M}$, onde $\mathrm{M}=\left\{\left(\rho_{i}, \rho_{j}\right) / \rho_{i} \leq_{l} \rho_{j}, i, j=\{1, \ldots, N !\}\right\}$.

$(\Leftarrow)$ Queremos provar que para dois nós quaisquer $\rho_{\mathrm{i}}$ e $\rho_{\mathrm{j}}$ em $\Pi_{\mathrm{N}}$, com $Z\left(\rho_{i}\right) \leq_{l} Z\left(\rho_{j}\right)$ e $\left|\mathfrak{T}\left(\rho_{j}\right)\right|-\left|\mathfrak{T}\left(\rho_{i}\right)\right|=m$, deve existir um caminho entre eles em que os $m$ arcos estão em W'. Suponhamos que não exista tal caminho, ou seja, para todo caminho entre $\rho_{\mathrm{i}}$ e $\rho_{\mathrm{j}}$, existe pelo menos um arco $\left(\rho_{\mathrm{i}}{ }^{\prime}, \rho_{\mathrm{j}}{ }^{\prime \prime}\right) \notin \mathrm{W}$ '. Sejam os caminhos parciais entre $\rho_{\mathrm{i}}$ e $\rho_{\mathrm{j}}, \mu_{1}=\left(\rho_{i}, \rho_{i}{ }^{\prime}, \rho_{i}{ }^{\prime \prime}\right)$ e $\mu_{2}=\left(\rho_{j}{ }^{\prime \prime}, \rho_{j}{ }^{\prime}, \rho_{j}\right)$, tais que $\left(\rho_{\mathrm{i}}, \rho_{\mathrm{i}}{ }^{\prime}\right) \in \mathrm{W}^{\prime}$ e $\left(\rho_{\mathrm{i}}{ }^{\prime}, \rho_{\mathrm{i}}{ }^{\prime}\right) \in \mathrm{W}^{\prime} ;\left(\rho_{\mathrm{j}}{ }^{\prime}, \rho_{\mathrm{j}}{ }^{\prime}\right) \in \mathrm{W}^{\prime}$ e $\left(\rho_{\mathrm{j}}{ }^{\prime}, \rho_{\mathrm{j}}\right) \in \mathrm{W}^{\prime}$. Segue-se do Lema 4.4 que

$$
Z\left(\rho_{i}\right) \leq_{l} Z\left(\rho_{i}^{\prime}\right) \leq_{l} Z\left(\rho_{i}^{\prime \prime}\right) \Rightarrow Z\left(\rho_{i}\right)-Z\left(\rho_{i}{ }^{\prime \prime}\right) \leq 0
$$

e

$$
Z\left(\rho_{j}{ }^{\prime \prime}\right) \leq_{l} Z\left(\rho_{j}{ }^{\prime}\right) \leq_{l} Z\left(\rho_{j}\right) \Rightarrow Z\left(\rho_{j}{ }^{\prime \prime}\right)-Z\left(\rho_{j}\right) \leq 0 .
$$

Somando-se ambos os membros das desigualdades provenientes de (16) e (17) chegamos a

$$
Z\left(\rho_{i}\right)-Z\left(\rho_{i}{ }^{\prime \prime}\right)+Z\left(\rho_{j}{ }^{\prime \prime}\right)-Z\left(\rho_{j}\right) \leq 0 .
$$

Como $\left(\rho_{\mathrm{i}}{ }^{\prime}, \rho_{\mathrm{j}}{ }^{\prime \prime}\right) \notin \mathrm{W}$ ', tomemos um exemplar do PQA tal que $Z\left(\rho_{i}{ }^{\prime \prime}\right)>Z\left(\rho_{j}{ }^{\prime \prime}\right)$. Então é possível encontrar um $K>0$ e $K$ suficientemente grande, de modo que $Z\left(\rho_{i}{ }^{\prime \prime}\right)-Z\left(\rho_{j}{ }^{\prime \prime}\right)=K$. Somando-se este valor a equação (18) obtém

$$
Z\left(\rho_{i}\right)-Z\left(\rho_{i}^{\prime \prime}\right)+Z\left(\rho_{i}^{\prime \prime}\right)-Z\left(\rho_{j}{ }^{\prime \prime}\right)+Z\left(\rho_{j}{ }^{\prime \prime}\right)-Z\left(\rho_{j}\right) \geq 0 .
$$

A expressão (19) é simplesmente $Z\left(\rho_{i}\right)-Z\left(\rho_{j}\right) \geq 0$, contrariando o fato de $\left(\rho_{\mathrm{i}}, \rho_{\mathrm{j}}\right) \in \mathrm{M}$.

Como conseqüência do Teorema das Inversões, temos o seguinte: considere um par de permutações livremente comparáveis, em que cada elemento do par está situado em níveis distintos de $G_{L i v}$. Aquela que pertencente a um nível mais baixo no grafo tem custo não maior que a situada em nível superior.

\section{Avaliação do Parâmetro dado pelo Número de Inversões}

Três tipos de testes empíricos são apresentados na tentativa de validar o número de inversões como parâmetro de avaliação da qualidade de soluções de exemplares do PQA. O primeiro analisa em que altura do grafo $G_{L i v}$ se encontra a permutação $\rho$ correspondente à solução ótima $\varphi^{*}$ ou a melhor viável conhecida de cada caso testado. O segundo compara a curva da 
função custo com a descrita pelo número de inversões das respectivas permutações a eles associados; finalmente, o terceiro teste utiliza a técnica muito conhecida por análise de regressão, considerando como amostra um conjunto de permutações geradas aleatoriamente e tendo como variáveis seus respectivos números de inversões e custos.

\subsection{Melhores soluções conhecidas de exemplares PQA em $G_{\text {Liv }}$}

Seja $G_{L i v}=\left(\Pi_{\mathrm{N}}, \mathrm{M}\right)$ o grafo de comparabilidade do poset das permutações livremente comparáveis, cuja altura é $N(N-1) / 2+1$. Esta altura corresponde ao número máximo de inversões de para qualquer $\rho$ em $\Pi_{N}$. Chamamos de altura de uma permutação $\rho \in \Pi_{N} 0$ seguinte parâmetro:

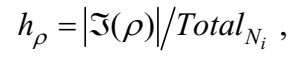

onde $/ \mathfrak{I}(\rho) /$ é o número de inversões de $\rho$ e $\operatorname{Total}_{N_{i}}=N(N-1) / 2+1$. Calculamos as alturas das permutações correspondentes às soluções ótimas ou melhores conhecidas dos problemas teste da QAPLIB [BKR97], listadas em 7 grupos na Tabela 1. Determinamos as médias das alturas para cada grupo de exemplares. Na Tabela 2, listamos tais médias, em colunas distintas, uma para as médias das soluções ótimas e outra para as de melhores soluções viáveis registradas na QAPLIB.

Tabela 1 - Grupos de caso da QAPLIB

\begin{tabular}{|l|l|l|l|}
\hline Exemplar & \multicolumn{1}{|c|}{ Dimensão } & Exemplar & \multicolumn{1}{c|}{ Dimensão } \\
\hline Scr & $n=12,15$ e 20 & Had & $n=12,15$ e 20 \\
\hline Chr & $n=12,15$ e 18 & Nug & $n=12,15$ e 20 \\
\hline Ste & $n=36$ & Rou & $n=12,15$ e 20 \\
\hline Sko & $n=42,49,64$ e 100 & & \\
\hline
\end{tabular}

Tabela 2 - Média das alturas por grupo de exemplares da QAPLIB

\begin{tabular}{|l|l|l|l|}
\hline $\begin{array}{c}\text { Grupo de casos com } \\
\text { solução ótima conhecida }\end{array}$ & Média & \multicolumn{1}{|c|}{$\begin{array}{c}\text { Grupo de casos com melhor } \\
\text { solução viável conhecida }\end{array}$} & Média \\
\hline Had & $31,00 \%$ & Ste 36 & $37,50 \%$ \\
\hline $\mathrm{Nug}$ & $31,05 \%$ & Sko $42,49,64$ & $38,98 \%$ \\
\hline Rou & $28,02 \%$ & Sko 100 & $41,00 \%$ \\
\hline Scr & $32,45 \%$ & --- & \\
\hline Chr & $35,85 \%$ & --- & $39,12 \%$ \\
\hline Média Total & $31,68 \%$ & Média Total & \\
\hline
\end{tabular}

A média total das alturas das médias dispostas na Tabela 2 para os grupos de casos do problema com ótimo conhecido fica em torno de $31,68 \%$ no grafo $G_{L i v}$, enquanto a média correspondente às alturas dos exemplares em que apenas conhecemos as melhores soluções é de $39,12 \%$. Este fato poderá indicar que para estes grupos de problemas podemos procurar 
soluções que possuam representantes com menor número de inversões. Destacamos o caso Sko100 do grupo Sko, pois a altura ficou em 41\%, o que indica que a solução viável encontrada deve estar um pouco mais longe da ótima. Este fato é bastante natural, dado que os exemplares de dimensão 100 são muito difíceis e os algoritmos heurísticos encontram boas soluções viáveis, porém com menos precisão.

\subsection{Gráficos de Comparação: Custos x Número de Inversões}

Para uma outra comparação entre os custos das soluções dos exemplares testados com o número de inversões das respectivas permutações no grafo $G_{L i v}$, traçamos gráficos de pares de curvas, uma referente aos custos de 50 soluções $\varphi \in \Pi_{n}$ do PQA, geradas para cada caso da Tabela 1 e outra referente ao número de inversões das respectivas permutações $\rho \in \Pi_{\mathrm{N}}$. A primeira curva é descrita pelos pares $\left(i, Z\left(\varphi_{i}\right)\right), i=1,2, \ldots 50$ tal que $Z\left(\varphi_{\mathrm{i}}\right)$ é o custo da solução $i$ e a segunda, pelos pares $\left(i, / \mathfrak{T}\left(\rho_{i}\right) /\right)$ com $\rho_{\mathrm{i}} \in \Pi_{\mathrm{N}}$. Como exemplo, escolhemos as curvas referentes às soluções geradas para Rou20 e Nug20. Mesmo não sendo livremente comparáveis todos os pares das 50 soluções geradas, os gráficos descrevem curvas muito semelhantes. As Figuras 1a e 1b apresentam os gráficos relacionados a Rou20 em que $92 \%$ dos pontos coincidem e as Figuras 2 a e 2 b ilustram Nug20, em que $86 \%$ dos pontos descrevem o mesmo traçado para as curvas. Para os outros casos, o comportamento foi semelhante, com exceção do grupo Chr (Christofides), que talvez seja o grupo dos casos mais difíceis de serem resolvidos, dentre aqueles registrados na literatura. Para exemplificar, Chr18a obteve apenas 50\% de coincidência no traçado.

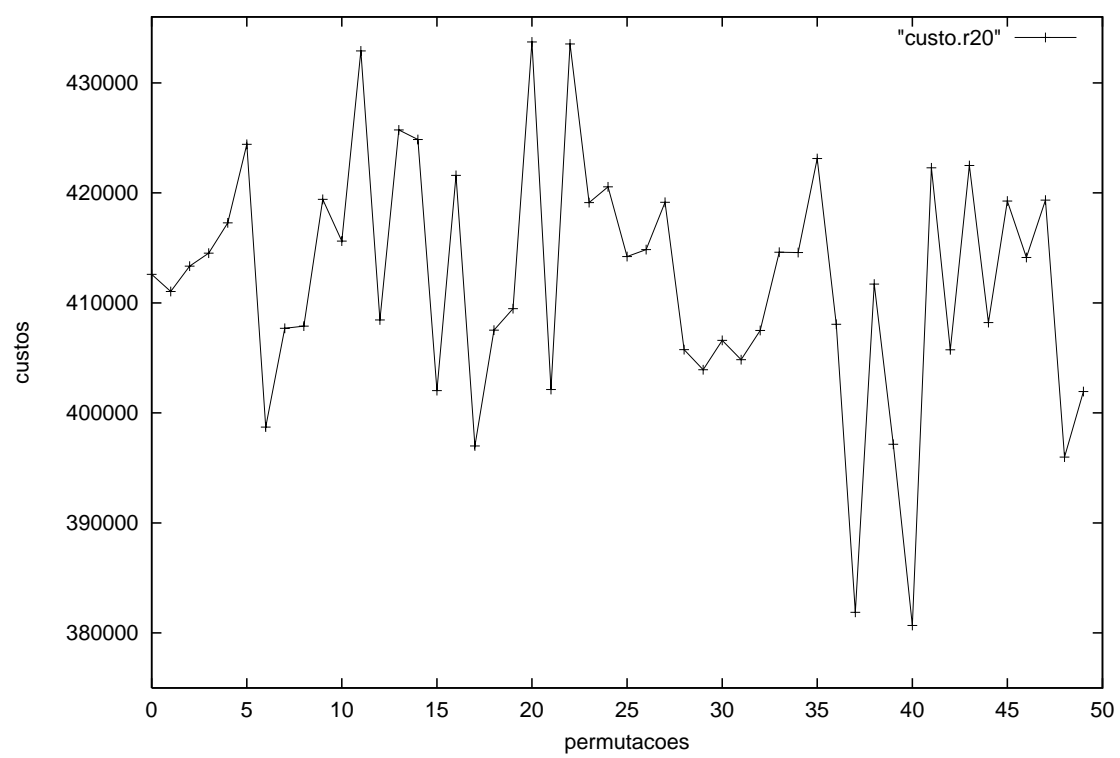

Figura 1a - Curva de variação dos custos - Rou20 


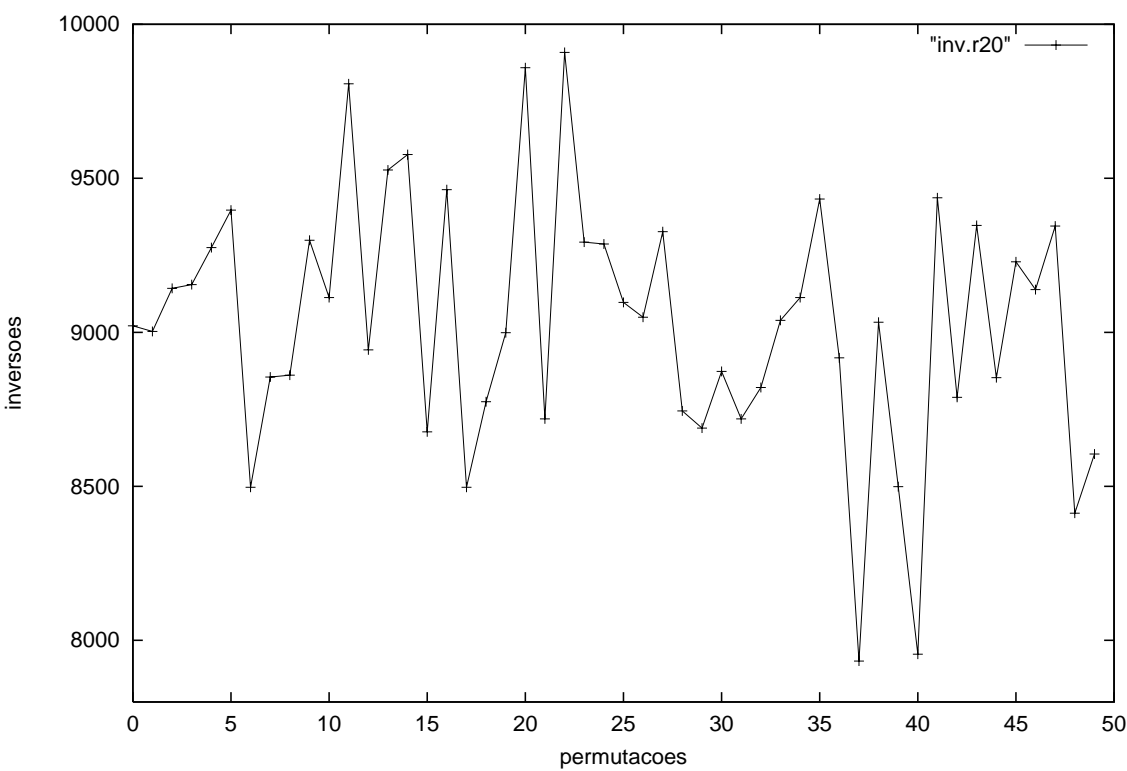

Figura 1b - Curva de variação das inversões - Rou20

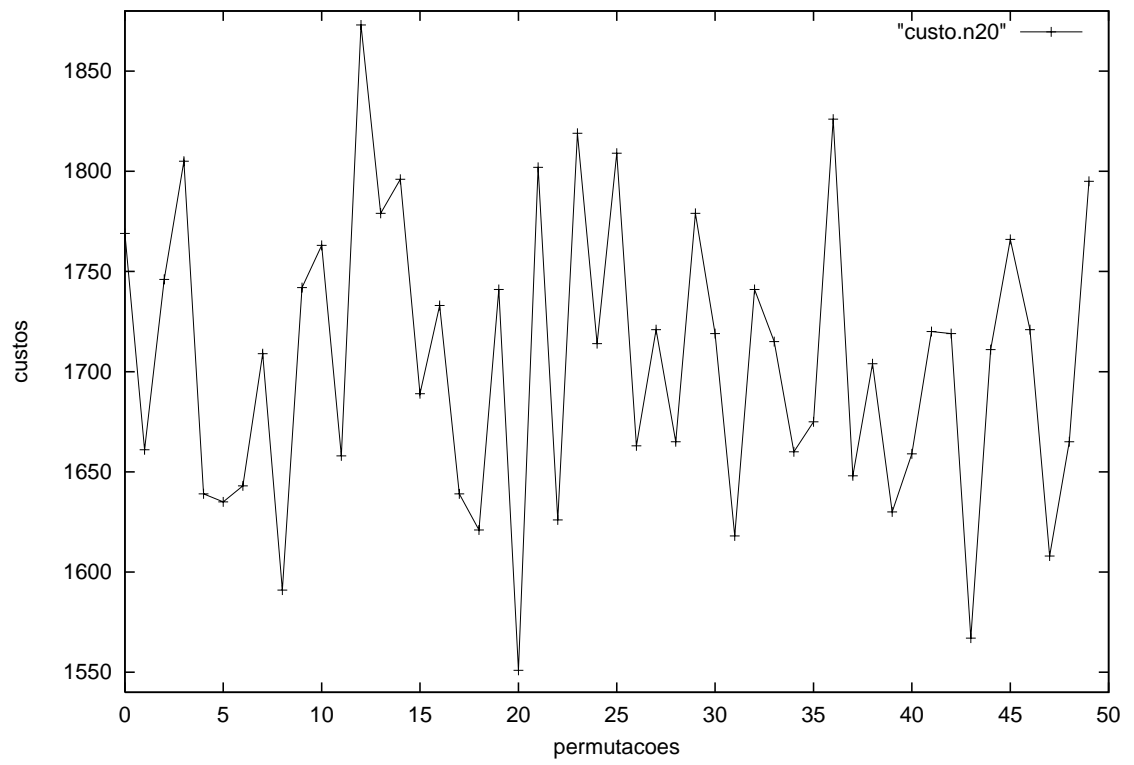

Figura 2a - Curva de variação do custo - Nug20 


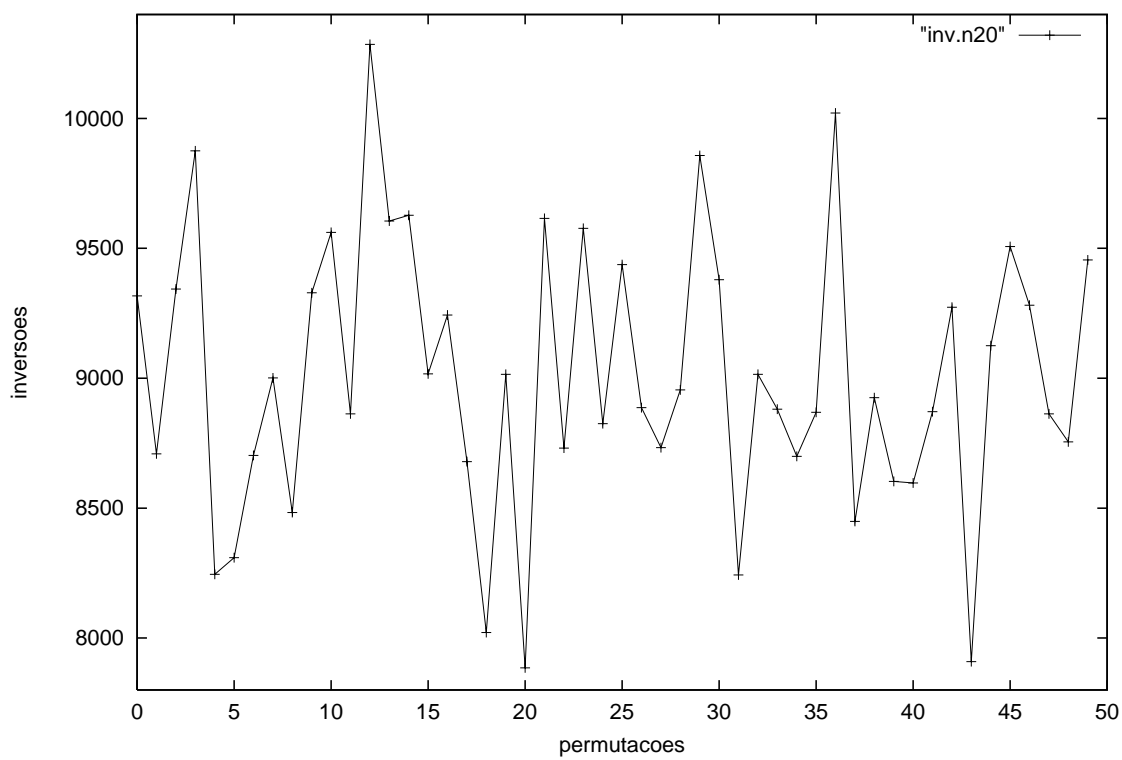

Figura 2b - Curva de variação das inversões - Nug20

\subsection{Análise por Regressão Linear}

Sabemos que um dos objetivos da análise por regressão linear é predizer o valor da variável dependente em função de um valor conhecido da variável independente. Para os testes que fizemos usando esta técnica, geramos uma amostra de 150 soluções de exemplares do PQA, para os quais calculamos custos e número de inversões das respectivas permutações. Os números de inversões foram tomados como variáveis livres e os custos como dependentes. Os diagramas de dispersão foram construídos com os números de inversões em ordem crescente sobre o eixo das abscissas e para $i=1,2 \ldots, 150$ marcamos os pontos $\left(\mid \mathfrak{I}\left(\rho_{i}\right) /, Z\left(\varphi_{i}\right)\right)$, no plano cartesiano. Os parâmetros da linha que fornece a melhor aproximação são estimados pelo Método dos Mínimos Quadrados que pode ser encontrado em qualquer referência básica de Estatística, como exemplo [CF76].

Em nossas amostras temos $k=150$, logo podemos aproximar a distribuição $t$-Student pela normal, que para um nível de significância $\alpha=0,05$, o parâmetro crítico é tabelado em $\mathrm{t}_{\mathrm{c}}=1,645$.

Felizmente todos os coeficientes lineares determinados das retas estimados pelo Método dos Mínimos Quadrados foram positivos, indicando a relação direta entre custos e número de inversões, cujos testes de hipóteses validaram a relação linear e direta, a exceção de Chr18b. Como exemplo, escolhemos novamente Rou20 e Nug20. As Figuras 3 e 4 mostram as representações gráficas das retas de regressão, para cada caso, cujas relações entre custo $\mathbf{x}$ número de inversões estão bem próximas, dado que ambos os coeficientes de correlação $r$ são próximos a 1. Para os outros exemplares dos grupos das Tabelas 1 e 2, o comportamento foi semelhante, a exceção de Ste36a e Ste36b, cujos coeficientes de correlação estão próximos a 0,25. Mesmo assim, os testes de hipóteses confirmam a relação linear e direta. 


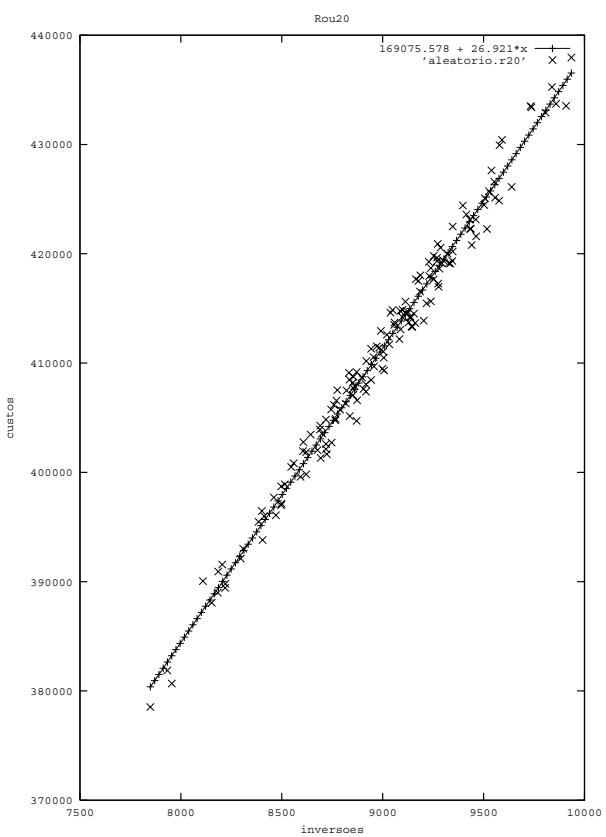

Figura 3 - Reta de regressão $y=169075,6+26,9 x \operatorname{com} r=0,9852$ para Rou20.

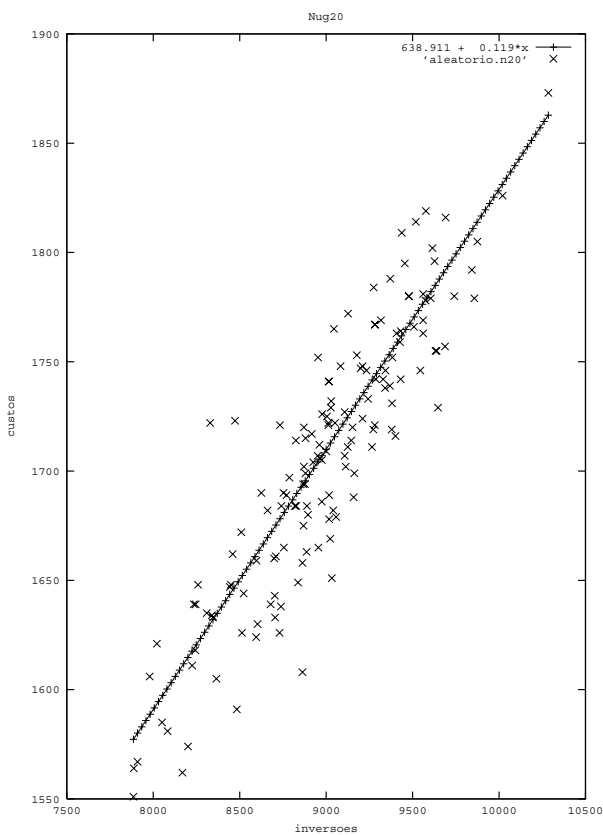

Figura 4 - Reta de regressão com a expressão y $=638,9+0,12 x \operatorname{com} r=0,8073$ para Nug20. 


\section{Conclusões}

Neste trabalho provamos dois teoremas: o Teorema da Ordenação Parcial Livre e o Teorema das Inversões. O primeiro estabelece uma ordenação no conjunto de soluções do PAL e conseqüentemente no conjunto de soluções dos exemplares do PQA a ele relacionados. Tal ordenação induz uma nova ordenação no conjunto das permutações que contém a conhecida ordenação dada pelas inversões das permutações, vértices do grafo $G_{L i v}$. Isto permitiu demonstrar, utilizando grafos de comparabilidade, o segundo teorema. Este nos deu a possibilidade de investigar se o número de inversões correspondentes às soluções de exemplares do PQA pode ser usado como parâmetro para avaliação da qualidade das soluções encontradas por técnicas heurísticas, já que as mesmas não garantem a otimalidade da solução encontrada. Assim, fizemos três tipos de testes na tentativa de validação do referido parâmetro. No primeiro teste, concluímos que a solução ótima de qualquer caso do problema se encontra, em média a $32 \%$ da altura do grafo $G_{L i v}$ e para os exemplares que ainda não foram resolvidos otimamente tem-se para as melhores soluções viáveis conhecidas uma altura, em média, $39 \%$ do grafo. O fato de a primeira média ser menor que a segunda é perfeitamente aceitável, pois acreditamos que as soluções ótimas desses casos, que ainda não se sabe se foram ou não encontradas, possuam representantes em $G_{L i v}$ com menor número de inversões. Fizemos o segundo teste por comparação das curvas que descrevem os comportamentos dos custos das soluções e dos números de inversões das permutações correspondentes em $G_{L i v}$, e observamos que, em média, $76 \%$ dos pontos desenhados apresentam a mesma trajetória. O terceiro e último teste, correspondente à análise de regressão linear, mostra que os custos estão diretamente relacionados com o número das inversões das permutações associadas, dado que encontramos os coeficientes da reta sempre positivos e significativamente diferentes de zero. Pelo menos para os pares de permutações livremente comparáveis, o Teorema das Inversões já estabelece que seus correspondentes custos crescem com os números de inversões das suas respectivas permutações. Verificamos, através dos testes, que até mesmo em relação a pares de permutações não livremente comparáveis, é possível manter esta tendência para a maioria dos casos. Cabe acrescentar que o resultado provado pelo Teorema das Inversões havia sido proposto por [Ab84] e já foi aplicado na definição de movimentos de buscas locais utilizadas pelas metaheurísitcas GRASP, [RAB00] e Simulated Annealing [AQB99], ambos apresentando resultados satisfatórios para o PQA. Também vale à pena acrescentar que o Teorema da Ordenação Parcial Livre, além de estabelecer uma ordenação no conjunto das soluções dos problemas aqui estudados, certamente nos permite estabelecer critérios hierárquicos de decisão na escolha das soluções num prosseguimento da busca local. Este teorema introduz uma ordenação parcial no conjunto das permutações baseada em produtos escalares, caracterizando um poset que contém o já conhecido poset das inversões, cujo diagrama de Hasse determina o permutaedro [RA02]. Este último fato é uma contribuição à Matemática Discreta.

\section{Agradecimentos}

Os autores agradecem ao CNPq pelo apoio a esta pesquisa através do projeto PROTEM-CC. 


\section{Referências Bibliográficas}

[Ab84] Abreu, N.M.M. (1984). Um estudo algébrico e combinatório do problema quadrático de alocação segundo Koopmans e Beckmann. Tese de D.Sc. Programa de Engenharia de Produção - COPPE/UFRJ, Brasil.

[ABLG02] Anstreicher, K.M.; Brixius, N.W.; Linderoth, J. \& Goux, J.P. (2002). Solving Large Quadratic Assignment Problems on Computational Grids. Mathematical Programming, Series B, 91, 563-588. Winner, SIAM Activity Group on Optimization (SIAG/OPT) Prize.

[ABQG02] Abreu, N.M.M.; Boaventura-Netto, P.O.; Querido, T.M. \& Gouvêa, E.F. (2002). Classes of quadratic assignment problem, instances: isomorphism and difficulty measure using a statistical approach. Discrete Applied Mathematics, 124, 103-116, Elsevier Science Inc.

[AQB99] Abreu, N.M.M.; Boaventura-Netto, P.O. \& Querido, T.M. (1999). REDINV-SA: A Simulated Annealing for the Quadratic Assignment Problem. RAIRO Operations Research, 33, 249-273.

[An01] Anstreicher, K.M. (2001). Eigenvalue Bounds Versus Semidefinite Relaxations for the Quadratic Assignment Problem. SIAM Journal on Optimization, 11, 254-265.

[AZ02] Angel, E. \& Zissimopoulos, V. (2002). On the Hardness of the Quadratic Assignment Problem with Metaheuristics. Journal of Heuristics, 8, 399-414.

[BKR97] Burkard, R.E.; Karisch, S.E. \& Rendl, F. (1997). QAPLIB - A Quadratic Assignment Problem Library. Journal of Global Optimization, 10, 391-403. Acesso internet: http://www.opt.math.tu-graz.ac.at/qaplib/

[Çe98] Çela, E. (1998). The Quadratic Assignment Problem - theory and algorithms. Series in Combinatorial Optimization, 1, Kluwer Academic Publishers.

[CF76] Crusius, C.A. \& Fachel, J.M. (1976). Estatística aplicada à economia e administração. McGraw-Hill.

[Co90] Connolly, D.T. (1990). An improved annealing scheme for the QAP. European Journal Operations Research, 46, 93-100.

[Ed77] Edwards, C.S. (1977). The derivation of a greedy approximated for the KoopmansBeckmann quadratic assignment problem. In: Proceedings of the 77-th Combinatorial Programming Conference, 55-86.

[Ed80] Edwards, C.S. (1980). A branch and bound algorithm for the Koopmans-Beckmann quadratic assignment problem. Mathematical Programming Study, 13, 35-52.

[FY83] Frieze, A.M. \& Yadegar, J. (1983). On the quadratic assignment problem. Discrete Applied Mathematic, 5, 89-98.

[GG02] Goldbarg, M.C. \& Goldbarg, E.F.G. (2002). Transgenética Computacional: uma aplicação ao problema quadrático de alocação. Pesquisa Operacional, 22, 359-386.

[Gi62] Gilmore, P.C. (1962). Optimal and suboptimal algorithms for the quadratic assignment problem. SIAM Journal on Applied Mathematics, 10, 305-313.

[GTD99] Gambardella, L.M.; Taillard, É.D. \& Dorigo, M. (1999). Ant Colonies for the Quadratic Assignment Problems. Journal of the Operational Research Society, 50, 167-176. 
[HLP52] Hardy, G.H.; Littlewood, J.E. \& Pólya, G. (1952). Inequalities. Cambridge University Press.

[KB57] Koopmans, T.C. \& Beckmann, M.J. (1957). Assignment problems and the location of economics activities. Econometrica, 25, 53-76.

[KB78] Kaufman, L. \& Broeckx, F. (1978). An algorithm for the quadratic assignment problem using Bender's decomposition. European Journal Operational Research, 2, 204-211.

[La63] Lawler, E.L. (1963). The quadratic assignment problem. Management Science, 9, 586-589.

[LPR94] Li, Y.; Pardalos, P.M. \& Resende, M.G.C. (1994). A greedy randomized adaptive search procedures for the quadratic assignment problem. DIMACS Series in Discrete Mathematics and Theoretical Computer Science, 16, 237-261.

[Mi03] Misevicius, A. (2003). A modified tabu search algorithm for the quadratic assignment problem. Working Paper, Kaunas University of Technology, Kaunas, Lithuania.

[NVR68] Nugent, C.E.; Vollmannm T.E. \& Ruml, J. (1968). An experimental comparison of techniques for assignment of facilities to locations. Operations Research Quarterly, 16, 150-173.

[PR96] Padberg, M.W. \& Rijal, M.P. (1996). Location, Scheduling, Design and Integer Programming. Kluwer Academic Publishers, Boston.

[Ra00] Rangel, M.C. (2000). Contribuições algébricas para o problema quadrático de alocação. Tese de D.Sc. Programa de Engenharia de Produção - COPPE/UFRJ, Brasil.

[RA01] Rangel, M.C. \& Abreu, N.M.M. (2001). Um parâmetro para avaliar a qualidade das soluções do problema quadrático de alocação. In: CD-ROM XXXIII SBPO, Sobrapo, ILTC, 1, 1277-1287.

[RA02] Rangel, M.C. \& Abreu, N.M.M. (2002). Free poset involving permutations and scalar products: modeling LAP- and QAP-solution. Submetido a Elsevier Science Inc. em Novembro de 2002.

[RAB00] Rangel, M.C.; Abreu, N.M.M. \& Boaventura-Netto, P.O. (2000). GRASP para o PQA: um limite de aceitação para soluções iniciais. Pesquisa Operacional, 20, 45-58.

[Ta91] Taillard, É.D. (1991). Robust tabu search for the quadratic assignment problem. Parallel Computer, 17, 443-455.

[VRA95] Vernet, O.; Rodrigues, R.M.N.D. \& Abreu, N.M.M. (1995). Reticulados de Permutações. Relatório Técnico, EP-03/95 Série P.O., Programa de Engenharia de Produção - COPPE/UFRJ, Brasil.

[Zh96] Zhao, Q. (1996). Semidefinite programming for assignment and partition problems. Ph.D. Thesis, University of Waterloo, Ontario, Canada.

[ZKRW98] Zhao, Q.; Karisch, S.E.; Rendl, F. \& Wolkowicz, H. (1998). Semidefinite relaxations for the quadratic assignment problem. Journal Combinatorial Optimization, 2, 71-79. 